\title{
Analysis of Healthcare Provider Associated Barriers to Using Pre-Exposure Prophylaxis Medications to Prevent Human Immunodeficiency Virus Infections in the United States
}

\author{
Anderson JJ ${ }^{1}$, Vandergon $\mathrm{J}^{2}$ and Bastianelli \\ KMS $^{1 *}$ \\ ${ }^{1}$ Department of Pharmacy Practice and Pharmaceutical \\ Science, University of Minnesota College of Pharmacy, \\ USA \\ ${ }^{2}$ Positive Care Center at Hennepin, USA \\ *Correspondling author: Karen MS Bastianelli, \\ Department of Pharmacy Practice and Pharmaceutical \\ Sciences, University of Minnesota College of Pharmacy, \\ USA
}

Received: January 14, 2017; Accepted: March 03, 2017; Published: March 06, 2017

\begin{abstract}
Objective: Human Immunodeficiency Virus infections occur at high rates within at-risk populations in the United States. Men who have sex with men continue to bear the burden of new infections, with nearly $70 \%$ of new cases being transmitted through MSM contact, despite estimates of only $4 \%$ of the total population. A fixed dose combination of the antiretroviral drugs tenofovir and emtricitabine, was approved for use for pre-exposure prophylaxis (PrEP) in 2012. This paper looks to identify healthcare provider associated barriers to
\end{abstract} care.

Methods: A literature review using PubMed, Academic Search Premier, Ovid, and Google Scholar to identify peer-reviewed journals published between 2010 and present.

Results: Adherence, costs, antiviral resistance, perception of patient behavior and efficacy were barriers that were identified consistently throughout the research.

Discussion: The results reflect a need for additional education and training on behalf of healthcare providers. Identification and education to address these barriers will provide an opportunity for PrEP to become a more useful and utilized first line pharmacological therapy.

Keywords: Human immunodeficiency virus; World health organization; Pre-exposure prophylaxis; Food and drug administration

\section{Introduction}

\section{Epidemiology}

Human Immunodeficiency Virus (HIV) has played a significant role in healthcare within the United States and around the world since the early 1980's. The World Health Organization (WHO) estimates there are 36.7 million people worldwide living with HIV, 2.1 million who were infected in 2015 alone [1]. The Centers for Disease control estimates that there were nearly 40,000 new infections within the United States in 2015 and that 1.2 million people currently are living with HIV; moreover, 1 in 8 (12.8\%) are not aware of their status [2]. Despite an estimated $\$ 25$ billion annual budget for domestic response to HIV within the United States [3], some groups continue to show an increase in new infections.

Men who have sex with men (MSM) continue to bear the burden of new infections, with nearly $70 \%$ of new cases being transmitted through MSM contact, despite estimates of comprising only $4 \%$ of the total population [4,5]. For 2014, nearly 30,000 new infections can be attributed to MSM and reflect a $21 \%$ increase compared to 2008 new infections $[4,5]$. Specific populations within the MSM grouping that are disproportionally affected include Black/African Americans (38\%) accounting for the largest number of new infections among
MSM, despite the fact that African Americans only account for roughly $12 \%$ of the population [4]. Furthermore, nearly $20 \%$ of the new infections affect youths ages 13-24; consequently all tools to help prevent the spread of HIV need to be considered [4].

Tenofovir and emtricitabine combination formulation was approved by the Food and Drug Administration (FDA) in 2004 for use in initial treatment plans of newly infected HIV patients. It is considered first line therapy agent for initiation of antiretroviral therapy in treatment naïve patients [7]. In July 2012, the FDA approved it for prophylactic use to prevent HIV infections. It should be noted that the press release specifically stated "Truvada in combination with safe sex practices for Pre-Exposure Prophylaxis (PrEP) to reduce the risk of sexually acquired HIV-1 in adults at high risk" highlighting that the use of PrEP should not be a replacement for other forms of prophylaxis [8].

The approval came on the heels of high profile clinical trials studying the safety and effectiveness of the drug used prophylactic ally. The pre-exposure prophylaxis initiative (iPrEx) trial found that new HIV infections rate in HIV-negative gay men was reduced by $44 \%$ when given the two drugs (tenofovir and emtricitabine) compared to placebo9. Moreover, the efficacy of the combination drug therapy 
Table 1: Summary of 2014 CDC Guidelines section.

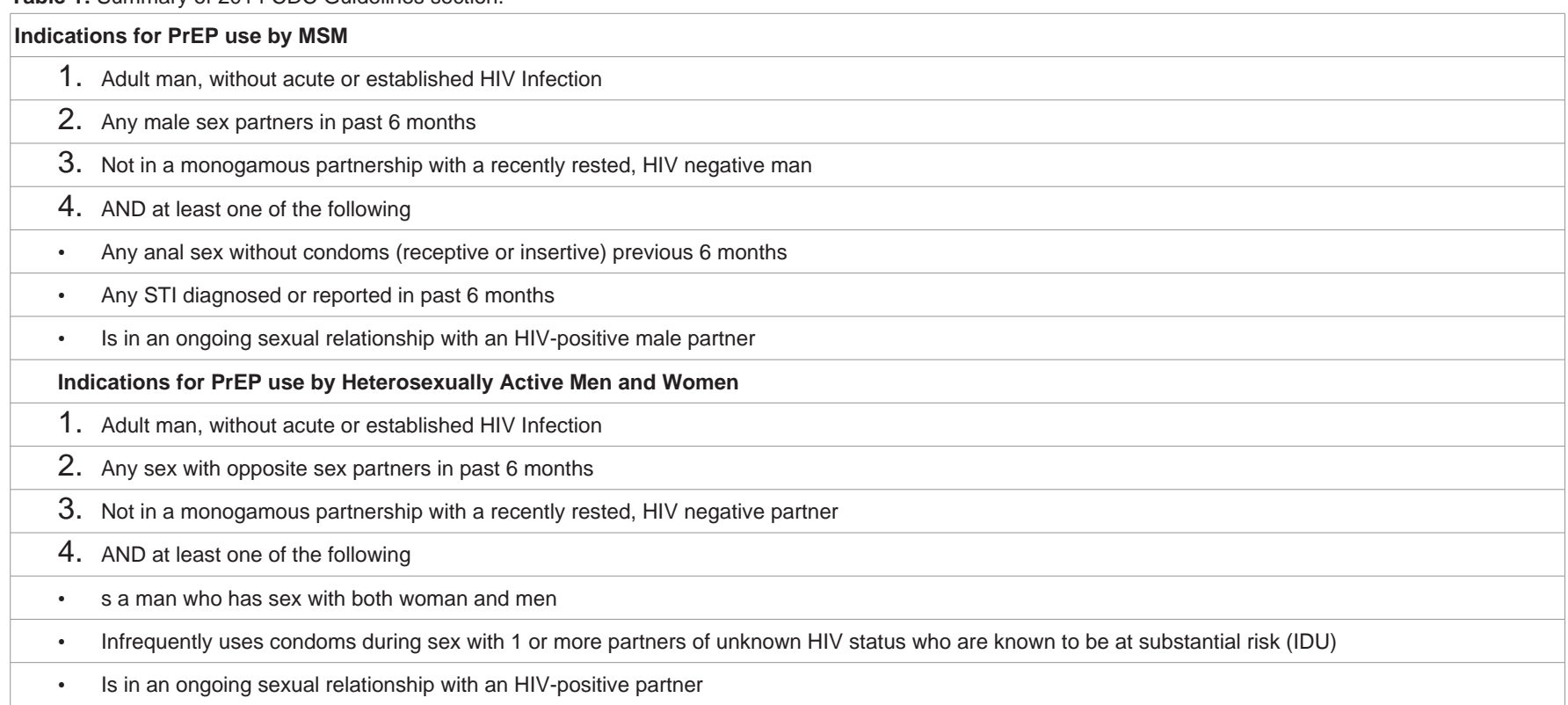

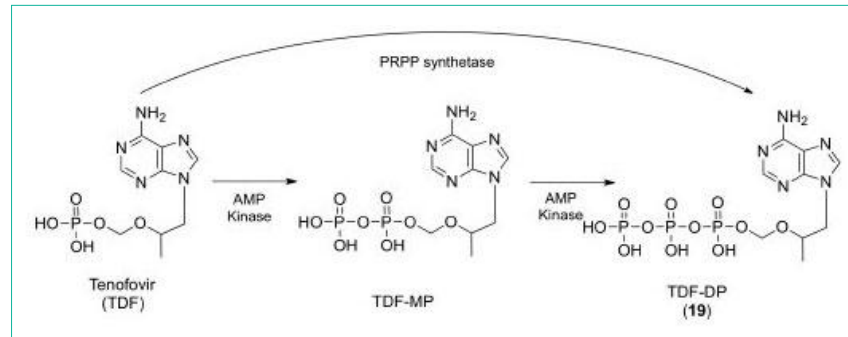

Picture 1: PRPP symthetase [15].

increased to $73 \%$ in patients who took the drugs more than $90 \%$ of the time [9]. An important note to this study reflects that although $93 \%$ of the patients reported to taking the medication correctly, druglevel monitoring in blood accounted for only $51 \%$ actually doing so [9]. In addition, only 3 out of $34(\sim 9 \%)$ of the subjects who became infected had drug levels that were detectable in the blood or cells [9]. In an extension to this study, the iPreExOLE (Open Label Extension) showed even more promising results, specifically as it relates to adherence and efficacy. While the group taking PrEP had half as many HIV infections compared to the placebo group, efficacy increased as number of doses per week increased. In patients who took 2-3 doses a week, the efficacy increased to $84 \%$ while they saw no new infections in patients who took at least four doses a week [10]. Modeling work completed on the data gathered from these studies provides close to $100 \%$ protection of the HIV Virus when PrEP is taken every day [10]. Additionally, a Cochrane Database System Review published in 2012, found a $51 \%$ decrease in risk for contracting HIV among high-risk individuals when taking tenofovir/emtricitabine or tenofovir alone compared to placebo. (RR 0.51; 95\% CI 0.30 to 0.86 ) [11]. Finally, results from a recently published study of men within the Kaiser Permanente healthcare system found no new HIV infections in the 657 members who used PrEP between 2012 and 2015 prophylactically [11].

Following the release of several clinical studies that measured the safety of PrEP, the US Public Health Service released the first comprehensive guidelines for PrEP in May 2014. The new guidelines provided clarification on identification of at-risk populations, as well as providing guidelines for prescribing and monitoring the therapy. These guidelines "recommend that PrEP be considered for people who are HIV-negative and at a substantial risk for HIV" [12]. The high-risk groups can be found in Table 1 within the Summary of 2014 CDC Guidelines section. Despite the recommendations, the number of patients being prescribed PrEP is thought to be extremely low. A 2014 survey completed by the Kaiser Family Foundation measured the attitudes and knowledge of gay men in the U.S. as it related to HIV therapies. Eight out of ten responders said they have heard little or nothing at all about PrEP, and only one out of ten responders knew someone who has taken PrEP [13]. A survey of physician members of the Infectious Diseases Society of America's (ISDA) Emerging Infections Network indicated that only $9 \%$ have actually prescribed PrEP [23]. While it never has been considered a replacement for safe sex practices, substantial debate regarding the use of PrEP has ensued. Understanding what barriers exist within the at-risks groups will provide an opportunity to utilize the therapy going forward.

\section{Pharmacology of tenofovir/emtricitabine}

Understanding the specific properties and pharmacology of the medication is a factor that needs to be considered when thinking about potential barriers for care. Tenofovir is a nucleotide analog HIV-1 reverse transcriptase inhibitor and an HBV reverse transcriptase inhibitor [14]. It is an acyclic analog of adenosine monophosphate. Tenofovir Disoproxil Fumurate (TDF) is the prodrug that is provided in the formulation. Upon hydrolysis and subsequent phosphorylation, tenofovir diphosphate is formed [14] as reflected in Picture 1 [15].

Tenofovir acts as a chain terminator by competing with a substrate, deoxyadenosine 5'-triphosphate of the virus's DNA strand (Picture 2). As a nucleotide analog, it does not need initial intracellular phosphorylation, an important difference between tenofovir and emtricitabine [14].

Emtricitabine (FTC) is a nucleoside analog HIV-1 reserve 


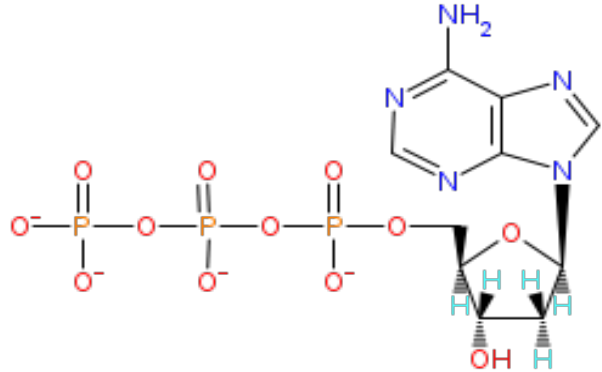

Picture 2: Deoxyadenosine 5'-triphosphate [16].

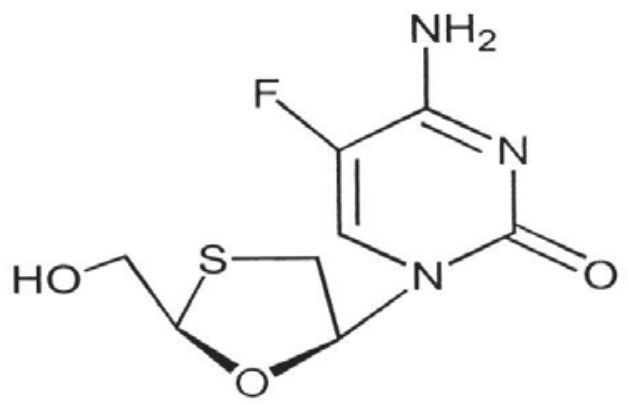

Picture 3: Emtricitabine [17].<smiles>Nc1ccn(C2CC(O)C(COP(=O)([O-])OP(=O)([O-])OP(=O)([O-])O)O2)c(=O)n1</smiles>

Picture 4: Deoxycytidine 5'-triphosphate [18].

transcriptase inhibitor (Picture 3). As an analog of cytidine, it inhibits HIV-1 transcriptase by competing with a different substrate of the virus, deoxycytidine 5'-triphosphate (Picture 4), incorporating itself into the nascent viral DNA and subsequent chain termination [14].

These processes prevent the replication of the virus within the host, i.e. once someone becomes infected. With PrEP, it is thought that combination product blocks HIV replication from occurring and establishing infection in the body. The CDC PrEP guidelines state "If you take Truvada as PrEP daily, the presence of the medication in your bloodstream can sometimes stop the virus from establishing itself and slow the spread of HIV in your body" [19]. The virus itself is constantly replicating and mutations can occur causing potential resistance. If the virus is blocked prior to establishing the infection in the body, the risk of resistance is mitigated. The half-life of tenofovir and emtricitabine are 17 hours and 10 hours respectively, an important consideration as it relates to adherence [14]. Finally, the side effects profile needs to be considered. Common side effects
Table 2: Recommendations for Clinicians Prescribing PrEP.

\section{Recommendations for Clinicians Prescribing PrEP}

1. Prescribe medication regimens based on risk assessment

2. Proper medication adherence education to maintain protective levels

3. Medication education to maximize safe use

4. HIV risk-reduction support and prevention service and/or referrals

5. Continual monitoring for toxicities, new HIV infections and on-going risk

6. TDF $300 \mathrm{mg}$ plus FTC $200 \mathrm{mg}$ once daily in MSM (Combination dose and once daily regimen only recommended dose/regimen for MSM)

Table 3: Clinical Follow-Up and Monitoring.

Clinical Follow-Up and Monitoring

At least every 3 months:

1. Repeat HIV testing to document HIV status

2. Prescription and refill authorization for no more than 90 days (next HIV test)

3. Assess side effect, adherence, and risk behaviors

4. Continuing education on current PrEP literature and information At least every 6 months:

1. Monitor Creatinine Clearance $(\mathrm{CrCl})$

2. More frequently is comorbid conditions exist, i.e. hypertension

3. Increase in $\mathrm{SCr}$ not indication to withhold provided $\mathrm{CrCl}>60 \mathrm{ml} / \mathrm{min}$

4. $\mathrm{CrCl}<60 \mathrm{ml} / \mathrm{min}$ would indicate a consultation with nephrologist

5. STI Testing

At least every 12 months:

1. Evaluate the need to continue PrEP as a component of HIV prevention

Optional Assessments

1. Bone Health - not recommended

2. Therapeutic Drug Monitoring - not recommended

3. Lack of established blood concentration

4. Serum concentrations reflect only very recent doses (non-valid estimate of consistent adherence

of this medication include flatulence, bloating, rash, diarrhea, nausea, dizziness, headache, dream disorder, fatigue and depression. However, preliminary clinical studies report minimal side effects in patients taking PrEP [14]. Summary of 2014 CDC Guidelines [12] (Table 1,2,3).

\section{Methodology}

The design of this study was a literature review. The inclusion criteria contained studies published in peer-reviewed journals published between 2010-present written in the English language. Exclusion criteria included editorials and opinion pieces. A total of 44 articles were reviewed with 14 accepted based on the inclusion criteria and the content aligned with the purpose of the analysis. The articles selected were found using search engines PubMed, Academic Search Premier, Ovid, and Google Scholar. Terms included in the search for the literature review included: HIV, HIV barriers, PrEP, Truvada, PrEP barriers, HIV Statistics, LGBT, MSM, HIV at-risk, PrEP providers, and PrEP insurance.

\section{Results}

One survey of 573 physicians from the Infectious Diseases Society 


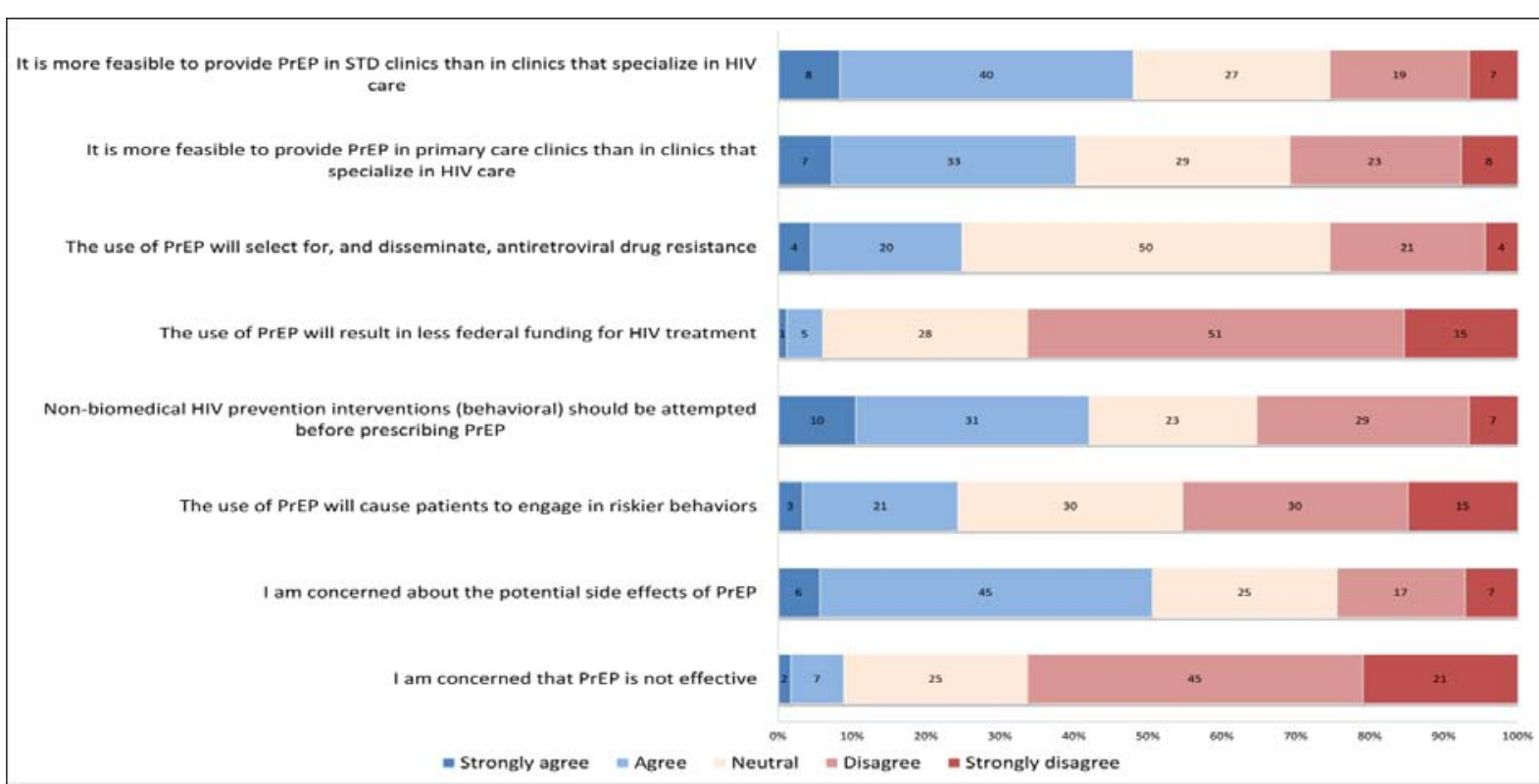

Figure 1: Clinician beliefs regarding pre-exposure prophylaxis [24].

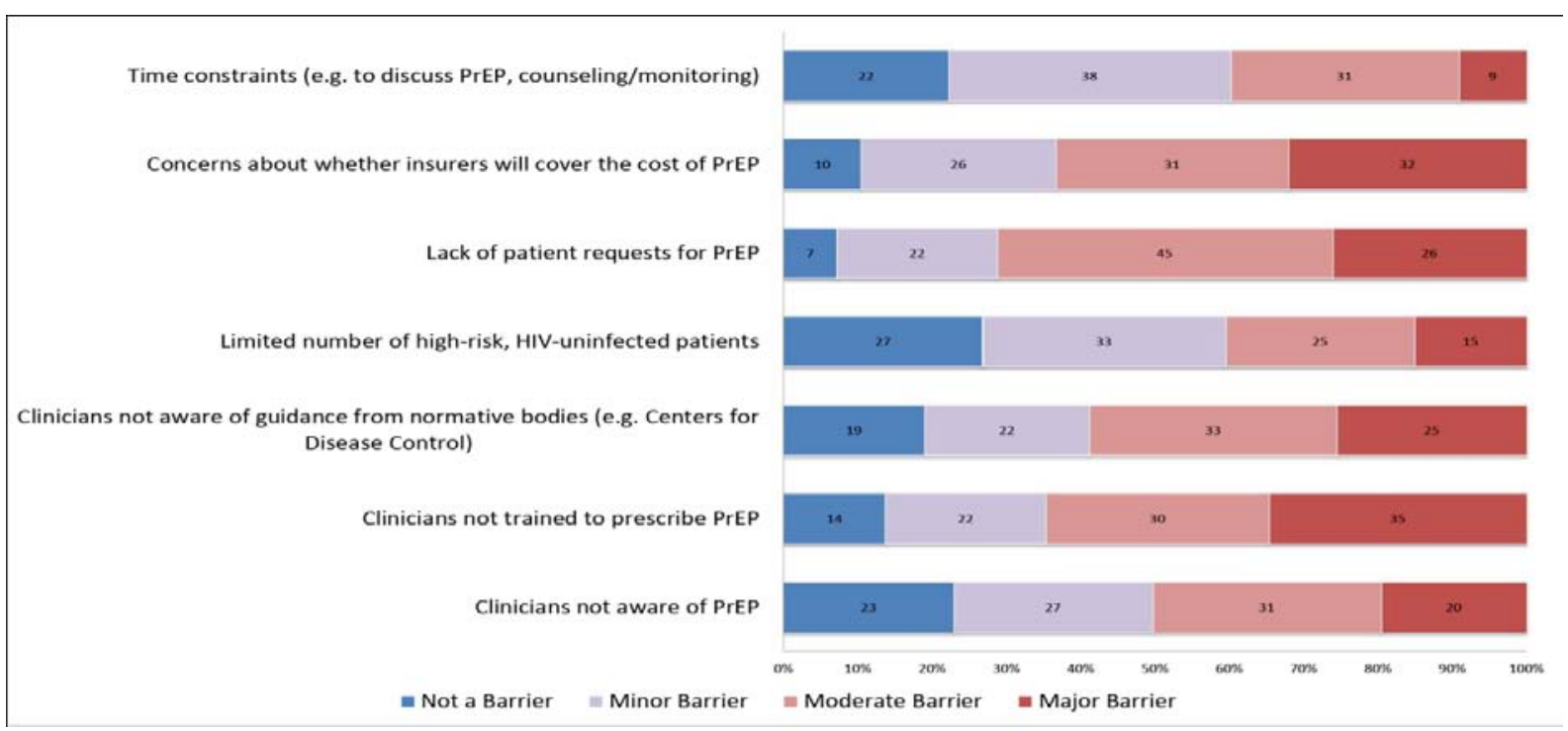

Figure 2: Clinician's perceived barriers to prescribing pre-exposure prophylaxis [24]

of America's (ISDA) Emerging Infections Network was completed assessing their attitudes towards PrEP. Seventy-four percent of the responders stated that they support the use of PrEP in at-risk individuals; twelve percent did not support PrEP, and fourteen percent unsure [20]. When asked whether they have provided PrEP, seventyseven responders stated they have not or would not provide PrEP. For this group, the most significant reason provided was concern about compliance and future resistance, with nearly $77 \%$ expressing concern poor compliance and the effect on future resistance [20]. Providers were allowed to mark more than one response and stated cost issues (57\%), toxicity (53\%), and insufficient evidence for efficacy in real world (53\%) were areas where more than half of this group provided reasons for not prescribing [20]. Additionally, these participants were allowed to mark "Other". Specifics reasons provided within the "Other" section include: "If they won't use condoms they won't use pills", "Creates a false sense of security", "Concern about irresponsible sexual activity", "Transmission can be prevented without medications" and "Medicine should not attempt to reverse bad behaviors" responses that indicate perceived risky behavior may impact prescribing practices [20].

The Karris study also asked all participants to rank the order of six specific barriers to provision of PrEP. The six specific barriers identified were time consuming to counsel and assess adherence, starting a potentially toxic drug in healthy persons, future drug resistance, too costly and patients can't afford it, patient population is not at risk for HIV infection, and concerns about efficacy of real- 
world PrEP. The cost of the drug ranked the highest among the six specific barriers that they prescribers were asked to rank [20]. The next two highest perceived barriers ranked by all participants were concern of future drug resistance and starting a toxic drug in a healthy person (something that was echoed by those stating they would or have not provided PrEP). Additionally, the participants were allowed to include comments to this question. Of particular note, responses include "Ours is an HIV clinic and not HIV negative care", "Is virtually a model for development and spread of resistant viruses", "PrEP needs to be in the primary care setting", and "It's an expensive condom" [20]. These challenges are not specific to the Karris study. An online study completed by 189 members of the American Academy of HIV medicine details similar concerns. Nearly one-third (32.3\%) of the responders listed development of viral resistance to antiretroviral as their greatest concern about prescribing PrEP, followed by adherence concerns (22\%) and high cost of drugs (21\%) [21]. Only $1.1 \%$ of participants stated they had no concerns about prescribing PrEP [21]

As was previously mentioned, new infection rates among minorities, especially African American and Latino's are increasing at an alarming rate. This could be an important consideration when assessing barriers as noted by a recent survey of medical studies in the northeast US. Of statistical significance, the future prescribers judge that black patients would be more likely to participate in risky behavior as a result of being on Prep compared to white patients, which in turn would impact their willingness to prescribe it [22] Additionally, in a survey of care providers in the southern US, race was also seen as a determinant, with prescribers less likely to prescribe to "other" race/ethnicity compared to white (aOR 0.23 ; 95\% CI 0.07 0.76.) [23].

Recently, more detailed surveys have delved into the beliefs and practices regarding the use of antiretroviral agents in the prevention of HIV spread. A survey of 184 healthcare providers in New England in 2013 regarding the use of antiretroviral medications included a substantial portion related to PrEP, which provided substantial data regarding the providers' beliefs regarding the use of PrEP, as well as their perceived barriers to prescribing PrEP, with the results shown in Figure 1 and Figure 2.

Only $19 \%$ of the clinicians surveyed had prescribed PrEP [24]. As Table 1 shows, the providers concern about potential side effects of PrEP was the response with the largest number of responders (61\%) marking either strongly agree or agree, while only $24 \%$ disagreeing with the concern over the potential side effects [24]. The next biggest concern related to who should be providing PrEP as $48 \%$ agreeing that it would be more feasible for PrEP in STD clinics than in clinics that specialize in HIV care compared to only $26 \%$ who disagreed [24]. 41\% agreed that non-biomedical HIV prevention interventions (behavioral) should be attempted before prescribing PrEP, while nearly $25 \%$ of responders believed that the use of PrEP will cause patients to engage in riskier behaviors as well as result in drug resistance [24]. Barriers identified by the providers that could impact patients included not being properly trained (65\%), concerns about insurers covering the cost of PrEP (63\%), as well as time constraints (40\%) [24].

Most recently, a study of providers from Washington DC and
Miami reflected practice-related perceived barriers in providing Prep to patients. The study identified two specifics groups of providers. One group tended to agree less when presented with statements that PrEP reduces the risk of HIV infections [25]. Half of this group expressed concern over the lack of time within their practice to provide PrEP [25]. Perceived patient behavior and history also tended to be a bigger concern with the group with a less favorable view of PrEP. The predicted probably that PrEP will promote risky behavior was twice as high in this group $\{(0.47[.36, .60], 95 \% \mathrm{CI})$ vs. $(0.24[.09, .49], 95 \% \mathrm{CI})\}$ [25]. These attitudes carried through when asked about the likelihood of prescribing PrEP to patient with perceived risky behavior. This group of providers were more than twice as less likely to prescribe PrEP to patients who had multiple sex partners $(20 \%$ vs. $43 \%, \mathrm{p}=0.4)$, more than three times less likely to prescribe PrEP to patients with a history of non-injection drug use ( $6.7 \% v s .23 .8 \%, \mathrm{p}=0.001)$, and more than twice as less likely to prescribe PrEP to patients with a history of failing to use condoms ( $16 \%$ vs. $36 \%, p=0.12)$ [25]. But it should be noted that risky behaviors may not be the only concern as this group's predicted probability of prescribing PrEP to serodiscordant couples was substantially lower $(0.67$ [.53,.77] $95 \%$ CI $)$ compared to the other group with a more positive view of $\operatorname{PrEP}(0.96[.69, .99]$ 95\% CI) [25]. Finally, resistance concerns were substantially higher in this group $\{(0.36[.26, .49], 95 \% \mathrm{CI})$ vs. $(0.23[.10, .45], 95 \% \mathrm{CI})\}[25]$.

\section{Discussion}

Across the various studies, adherence, future resistance, cost, safety, risky behaviors, and the lack of consensus on who should be providing PrEP were the most common barriers identified by the providers.

\section{Adherence and resistance}

In general, non-compliance is the largest reason for benefits not being realized from medications. Some estimates show up to $50 \%$ of patients do not take their medications properly [26]. With the potential side effects associated with anti-retroviral therapy, the concern for adherence is valid. The IprExOLE study reported the high level of efficacy in patients who took at least four doses per week, with zero new infections reported [10]. Adherence challenges were closely addressed in the iPreXOLE study. The higher the level of education, the higher the adherence was. In addition, adherence was substantially higher in participants who reported more than 5 sexual partners in 3 months, participants with a known HIV-positive partner, and participants who reported receptive anal sex without a condom [10]. These results show the importance for practitioners in assessing risk as it relates to adherence prior to prescribing PrEP.

The iPrEx study also considered the risk of resistance during the course of their study. While 10 subjects who were infected at the time of the enrollment, only 3 had resistance to FTC, with none of them showing TDF-resistance [9]. Of greater importance, 100 total subjects became infected during the course of the entire study, 36 in the group receiving PrEP, and 64 within the placebo. Zero subjects who became infected during the course of the study showed any resistance to FTC or TDF [9]. Additionally, the iPrEX study along with the CDC 4940 Trial showed that resistance to ART only occurred in those patients who were acutely infected but seronegative at the time the therapy was started [20]. 


\section{Cost}

A Google search completed on March 29, 2015 found that cash price for PrEP at retail locations in downtown Minneapolis is $\$ 1,300$ - \$1,600 for a one-month supply. This high cost certainly could be a burden on those without insurance and should be factored into a decision to prescribe PrEP. However, for those with insurance, there does not seem to be a significant cost or barrier to have private-insurers covering the cost of PrEP. Jim Pickett, the director of prevention at AIDS Foundation of Chicago reported that costs is often more affordable than clients expect [27]. Most insurance programs, including Medicaid, are now covering the cost of PrEP [28]. Additionally there is a substantial number of medication assistance and copay programs, such as the Gilead Co Pay program that offer assistance to patients with no insurance and to patients with insurance and high copays [29].

\section{Practice setting}

The debate about who should be prescribing PrEP is a valid concern. One could argue that the prescribing of PrEP should be done by HIV specialists who have more experience with these medications. However, some HIV specialists state the need for prophylactic treatment needs to be in the hands of primary care providers. This would require additional education training prior to prescribing these medications, something that primary care providers expressed the need for [30]. Educational and practice challenges were identified within the study that surveyed providers in the Washington DC and Miami areas. The group with the less favorable view of PrEP were also found to have less experience with PrEP, with nearly $90 \%$ of the responders having never prescribed it and nearly $96 \%$ of the responders working for organizations that lack a PrEP protocol [25]. This despite nearly $30 \%$ of this group of responders stating they had received requests for PrEP within the previous 6 months, and less than $10 \%$ of this group stating that had only seen $0-4$ patients with HIV in the practice within the previous 3 months of the survey [25]. When comparing this group with the group who described PrEP to be moderately or highly effective with few barriers, exposure and experience drove perceptions. The group who described PrEP to be moderately or highly effective in reduction of HIV infection risks were more likely to have worked in practices that had a PrEP protocol (23.8\% vs. $4.5 \%, \mathrm{p}=0.003)$, more likely to have received a request for PrEP within the previous 6 months (59.5\% vs. 29.2\%, $\mathrm{p}=0.004)$, and more likely to have prescribed PrEP at least once (36.6\% vs. $10 \%$, $\mathrm{p}<0.001)$ [25]. These results certainly indicate that with experience comes better practice behavior. But concerns regarding limitations of time were seen and echoed throughout several studies and need to be kept in mind when considering the barriers that the providers practice settings are adding to the mix.

\section{Risk behavior}

The views reflected in several of the studies pointing to concern of increased risky behavior or risk compensation may or may not have merit. Initial studies, including results from the IPreXOLE, show that patients on PrEP do not result in increased levels of risky behaviors [10]. The patients taking PrEP are motivated to prevent an infection and are more likely to have access to provision of condoms and counseling, through contact with prescribers. Additionally, the iPrEX trial reported no change in reported sexual behavior through follow- up [9]. There have been numerous other trials that have shown no increased in risk behavior or sexually transmitted diseases. And most recently, the PROUD Study Group produced results that showed no increase in risk behavior [32]. The importance of this finding was this was the first trial not using a placebo that failed to demonstrate any negative effect on risk behavior as it related to PrEP. However, the recently published Kaiser study completed in San Francisco, did report some potentially new behavior changes in patient were initiated PrEP. Of the 188 PrEP users asked about behavior changes after 6 months, $74 \%$ reported no change in the number of sexual partners, and $11 \%$ reported an increase in the number of sexual partners [32]. More concerning, $41 \%$ of the responders reported a decrease in condom use [32]. No statistically significant factors were identified with the decrease in condom use or increase in the number of partners. Additionally, it should be noted that no control group was used in this trial.

There are certainly limitations to this review of literature. The author relies on the research methods, statistical analysis, reporting of results, as well as the study questions posed in each survey. While several studies provided statistically significant results, some questions and results provided did not have statistically significance included in their results. Finally, several studies had limited number of participants.

In conclusion, the results from the literature review, including several studies, support the reality that providers are creating additional barriers to care in the use of tenofovir/emtricitabine for pre-exposure prophylaxis against HIV infections. Findings show that provider perceptions as it relates to patient behavior, cost, efficacy, safety, adherence, as well as practice setting are adding barriers. Being mindful of these barriers could provide an opportunity for state and federal agencies to better utilize resources when it comes to education and awareness for providers of PrEP. Finally, pharmacists can play a key role in providing education to allow for better utilization of the PrEP going forward.

\section{References}

1. Global Summary of the AIDS epidemic. 2015. World Health Organization.

2. HIV in the United States: At a Glance.

3. Global Health Policy. US Federal Funding for HIVIAIDS: Trends over Time.

4. Basic Statistics| Statistics Center | HIVIAIDS | CDC. Epidemiology of HIV Infection through 2014.

5. CDC Fact Sheet. Today's HIVIAIDS Epidemic. October 2016.

6. Minnesota Department of Health. HIVIAIDS Surveillance Reports. 2014.

7. FDA New Release. FDA approves first drug for reducing the risk of sexually acquired HIV infection. 2012.

8. AIDS info. Clinical Guidelines Portal. Guidelines for the use of Antiretroviral Agents in HIV-1 Infected Adults and Adolescents. What to Start: Initial Combination Regimens for the Antiretroviral-Naive Patient.

9. Grant RM, Lama JR, Anderson PL, McMahan V, Liu AY, Vargas L, et al. Pre exposure chemoprophylaxis for HIV prevention in men who have sex with men. N Engl J Med. 2010; 363: 2587-2599.

10. Grant RM, Anderson PL, McMahan V, Liu A, Amico KR, Mehrotra M, et al. Uptake of pre-exposure prophylaxis, sexual practices, and HIV incidence in men and transgender women who have sex with men: a cohort study. Lancet Infect Dis. 2014; 14: 820-829.

11. Volk JE, Marcus JL, Phengrasamy T, Blechinger D, Nguyen DP, Follansbee 
S, et al. No New HIV Infections with Increasing Use of HIV Pre exposure Prophylaxis in a Clinical Practice Setting. Clin Infect Dis. 2015.

12. US Centers for Disease Control. US Public Health Service. Report/Issue Brief. US Public Health Services Guidelines on Pre-Exposure Prophylaxis for the Prevention of HIV in the United States. May 2014

13. Hamel L, Firth J, Hoff T, Kates J, Levine S, Dawson L. HIVIAIDS in the Lives of Gay and Bisexual Men in the United States. The Henry J. Kaiser Family Foundation. 2014.

14. AIDSinfo Drug Data Base. Emtricitabine/Tenofovir Disoproxil Fumarate (Truvada) | FDA Label - Tablet (film coated).

15. Andrade $\mathrm{CH}$, de Freitas LM, de Oliveira V. Twenty-six years of HIV science: an overview of anti-HIV drugs metabolism. Braz. J. Pharm. Sci. 2011; 47: 209-230.

16. $\mathrm{GmbH}$, Molecular. 'Biopath-2-Prime-Deoxyadenosine-5-Prime-Triphosphate'. Molecular-networks.com. N.p., 2015. Web. 24 Nov. 2015.

17. Darque Albert, et al. Antimicrobial Agents and Chemotherapy. 2015.

18. Cobioshop.dk. 'Cobioshop.Dk - Dntps + Modified Dntps - Deoxycytidine 5-Triphosphate Disodium. N.p. 2015.

19. Truvada Medication Information Sheet - CDC PrEP Patient Fact Sheet.

20. Karris MY, Beekmann SE, Mehta SR, Anderson CM, Polgreen PM. Are we prepped for preexposure prophylaxis (PrEP)? Provider opinions on the realworld use of PrEP in the United States and Canada. Clin Infect Dis. 2014; 58: 704-712.

21. Tellalian D, Maznavi K, Bredeek UF, Hardy WD. Pre-exposure prophylaxis (PrEP) for HIV infection: results of a survey of HIV healthcare providers evaluating their knowledge, attitudes, and prescribing practices. AIDS Patient Care STDS. 2013; 27: 553-559.

22. Calabrese SK, Earnshaw VA, Underhill K, Hansen NB, Dovidio JF, et.al. The impact of patient race on clinical decisions related to prescribing HIV preexposure prophylaxis (PrEP): assumptions about sexual risk compensation and implications for access. AIDS Behav. 2014; 18: 226-240.
23. Tripathi A, Ogbuanu C, Monger M, Gibson JJ, Duffus WA.. Preexposure prophylaxis for HIV infection: healthcare providers' knowledge, perception, and willingness to adopt future implementation in the southern US. South Med J. 2012; 105: 199-206.

24. Krakower DS, Oldenburg CE, Mitty JA, Wilson IB, Kurth AE, Maloney KM, et.al. Knowledge, Beliefs and Practices Regarding Antiretroviral Medications for HIV Prevention: Results from a Survey of Healthcare Providers in New England. PLoS One. 2015; 10: e0132398.

25. Castel AD, Feaster DJ, Tang W, Willis S, Jordan H, Villamizar K, et.al. Understanding HIV Care Provider Attitudes Regarding Intentions to Prescribe PrEP. J Acquir Immune Defic Syndr. 2015.

26. Brown MT, Bussell JK. Medication Adherence: WHO Cares?. Mayo Clin Proc. 2011; 86: 304-314.

27. Heitz, D. Insurers and Medicaid Cover It. So What's Behind the Slow Adoption of Truvada PrEP?. 2014.

28. AIDS.gov. Pre-Exposure Prophylaxis (PrEP). US Department of Health and Human Services.

29. Fair Pricing Coalition. No dates listed. Medication Assistance Program and Co-Pay Programs for PrEP.

30. Krakower DS, Mayer KH. Pre-exposure prophylaxis to prevent HIV infection: current status, future opportunities and challenges. Drugs. 2015; 75: 243251.

31. Blumenthal, J, Haubrich R. Risk Compensation in PrEP: An Old Debate Emerges Yet Again. The Virtual Mentor: VM. 2014; 16: 909-915.

32. McCormack S, Dunn DT, Desai M, Dolling DI, Gafos M, Gilson R, et.al. Pre-exposure prophylaxis to prevent the acquisition of HIV-1 infection (PROUD): effectiveness results from the pilot phase of a pragmatic openlabel randomised trial.
Austin J Pharmacol Ther - Volume 5 Issue 1 - 2017 ISSN: 2373-6208 | www.austinpublishinggroup.com Bastianelli et al. (C) All rights are reserved
Citation: Anderson JJ, Vandergon J and Bastianelli KMS. Analysis of Healthcare Provider Associated Barriers to Using Pre-Exposure Prophylaxis Medications to Prevent Human Immunodeficiency Virus Infections in the United States. Austin J Pharmacol Ther. 2017; 5(1).1090. 